\title{
Virtual amalgamation of relatively quasiconvex subgroups
}

\author{
EDUARDO MARTÍNEZ-PEDROZA \\ AlESSANDRO Sisto
}

\begin{abstract}
For relatively hyperbolic groups, we investigate conditions guaranteeing that the subgroup generated by two relatively quasiconvex subgroups $Q_{1}$ and $Q_{2}$ is relatively quasiconvex and isomorphic to $Q_{1} * Q_{1} \cap Q_{2} Q_{2}$. The main theorem extends results for quasiconvex subgroups of word-hyperbolic groups, and results for discrete subgroups of isometries of hyperbolic spaces. An application on separability of double cosets of quasiconvex subgroups is included.
\end{abstract}

20F65, 20F67

\section{Introduction}

This paper continues the work started by the first author in [10] motivated by the following question:

Problem 1 Suppose $G$ is a relatively hyperbolic group and $Q_{1}$ and $Q_{2}$ are relatively quasiconvex subgroups of $G$. Investigate conditions guaranteeing that the natural homomorphism

$$
Q_{1} * Q_{1} \cap Q_{2} Q_{2} \longrightarrow G
$$

is injective and that its image $\left\langle Q_{1} \cup Q_{2}\right\rangle$ is relatively quasiconvex.

Let $G$ be a group hyperbolic relative to a finite collection of subgroups $\mathbb{P}$, and let dist be a proper left invariant metric on $G$.

Definition 1 Two subgroups $Q$ and $R$ of $G$ have compatible parabolic subgroups if for any maximal parabolic subgroup $P$ of $G$ either $Q \cap P<R \cap P$ or $R \cap P<Q \cap P$.

Theorem 2 For any pair of relatively quasiconvex subgroups $Q$ and $R$ of $G$ with compatible parabolic subgroups, and any finite index subgroup $H$ of $Q \cap R$, there is a constant $M=M(Q, R, H$, dist $) \geq 0$ with the following property. Suppose that $Q^{\prime}<Q$ and $R^{\prime}<R$ are subgroups such that:

(1) $H=Q^{\prime} \cap R^{\prime}$;

(2) $\operatorname{dist}(1, g) \geq M$ for any $g$ in $Q^{\prime} \backslash Q^{\prime} \cap R^{\prime}$ or $R^{\prime} \backslash Q^{\prime} \cap R^{\prime}$. 
Then the subgroup $\left\langle Q^{\prime} \cup R^{\prime}\right\rangle$ of $G$ satisfies:

(1) The natural homomorphism

$$
Q^{\prime} * Q^{\prime} \cap R^{\prime} R^{\prime} \longrightarrow\left\langle Q^{\prime} \cup R^{\prime}\right\rangle
$$

is an isomorphism.

(2) If $Q^{\prime}$ and $R^{\prime}$ are relatively quasiconvex, then so is $\left\langle Q^{\prime} \cup R^{\prime}\right\rangle$.

Theorem 2 extends results by Gitik [6, Theorem 1] for word-hyperbolic groups and by the first author [10, Theorem 1.1] for relatively hyperbolic groups. Yang recently obtained a similar combination results requiring stronger conditions [14]. His results include a combination result for HNN extensions and some applications to subgroup separability.

Definition 3 Two subgroups $Q$ and $R$ of a group $G$ can be virtually amalgamated if there are finite index subgroups $Q^{\prime}<Q$ and $R^{\prime}<R$ such that the natural map $Q^{\prime} * Q^{\prime} \cap R^{\prime} R^{\prime} \longrightarrow G$ is injective.

Let $Q$ and $R$ be relatively quasiconvex subgroups of $G$ with compatible parabolic subgroups and let $M=M(Q, R, Q \cap R)$ be the constant provided by Theorem 2. If $Q \cap R$ is a separable subgroup of $G$, then there is a finite index subgroup $G^{\prime}$ of $G$ containing $Q \cap R$ such that $\operatorname{dist}(1, g)>M$ for every $g \in G$ with $g \notin Q \cap R$. In this case, the subgroups $Q^{\prime}=G^{\prime} \cap Q$ and $R^{\prime}=G^{\prime} \cap R$ satisfy the hypothesis of Theorem 2; hence they have a quasiconvex virtual amalgam.

Corollary 4 (Virtual Quasiconvex Amalgam Theorem) Let $Q$ and $R$ be quasiconvex subgroups of $G$ with compatible parabolic subgroups, and suppose that $Q \cap R$ is separable. Then $Q$ and $R$ can be virtually amalgamated in $G$.

It is known that many (relatively) hyperbolic groups have the property that all quasiconvex or all finitely generated subgroups are separable; see Agol, Long and Reid [2], Long and Reid [8; 9], Wise [12; 13], and Agol, Groves and Manning [1]. Still, it is a natural question to ask whether the corollary above holds under the hypothesis that $G$ is residually finite.

A special case of the Virtual Quasiconvex Amalgam Theorem is the following by Baker and Cooper [3, Theorem 5.3].

Corollary 5 Let $G$ be a geometrically finite subgroup of isom $\left(\mathbb{H}^{n}\right)$, and let $Q$ and $R$ be geometrically finite subgroups of $G$ with compatible parabolic subgroups. Suppose $Q \cap R$ is separable in $G$. Then $Q$ and $R$ have a geometrically finite virtual amalgam. 
Separability of quasiconvex subgroups and double cosets of quasiconvex subgroups is of interest in the construction of actions on special cube complexes [13]. The machinery we use to prove the main result also gives the following.

Corollary 6 (Double cosets are separable) Let $G$ be a relatively hyperbolic group such that all its quasiconvex subgroups are separable. If $Q$ and $R$ are quasiconvex subgroups with compatible parabolic subgroups then the double coset $Q R$ is separable.

Acknowledgments We would like to thank Wen-yuan Yang for corrections on an earlier version of the paper, and for pointing out Corollary 6. We also thank the referee for insightful comments and corrections. Martínez-Pedroza is supported by the Natural Sciences and Engineering Research Council of Canada (NSERC).

\section{Preliminaries}

\subsection{Gromov-hyperbolic spaces}

Let $(X$, dist) be a proper and geodesic $\delta$-hyperbolic space. Recall that a $(\lambda, \mu)-$ quasigeodesic is a curve $\gamma:[a, b] \rightarrow X$ parameterized by arc length such that

$$
|x-y| / \lambda-\mu \leq \operatorname{dist}(\gamma(x), \gamma(y)) \leq \lambda|x-y|+\mu
$$

for all $x, y \in[a, b]$. The curve $\gamma$ is a $k$-local $(\lambda, \mu)$-quasigeodesic if the above condition is required only for $x, y \in[a, b]$ such that $|x-y| \leq k$.

Lemma 7 Coornaert, Delzant and Papadopoulos [5, Chapter 3, Theorem 1.2] (Morse Lemma) For each $\lambda, \mu, \delta$ there exists $k>0$ with the following property. In a $\delta$-hyperbolic geodesic space, any $(\lambda, \mu)$-quasigeodesic at $k$-Hausdorff distance from the geodesic between its endpoints.

Lemma 8 [5, Chapter 3, Theorem 1.4] For each $\lambda, \mu, \delta$ there exist $k, \lambda^{\prime}, \mu^{\prime}$ so that any $k$-local $(\lambda, \mu)$-quasigeodesic in a $\delta$-hyperbolic geodesic space is a $\left(\lambda^{\prime}, \mu^{\prime}\right)$ quasigeodesic.

Fix a basepoint $x_{0} \in X$. If $G$ is a subgroup of $\operatorname{Isom}(X)$, we identify each element $g$ of $G$ with the point $g x_{0}$ of $X$. For $g_{1}, g_{2} \in G$ denote by $\operatorname{dist}\left(g_{1}, g_{2}\right)$ the distance $\operatorname{dist}\left(g_{1} x_{0}, g_{2} x_{0}\right)$. Since $X$ is a proper space, if $G$ is a discrete subgroup of $\operatorname{Isom}(X)$, this is a proper and left invariant pseudometric on $G$. 
Lemma 9 [10, Lemma 4.2] (Bounded Intersection) Let $G$ be a discrete subgroup of isom $(X)$, let $Q$ and $R$ be subgroups of $G$, and let $\mu>0$ be a real number. Then there is a constant $M=M(Q, R, \mu) \geq 0$ so that

$$
Q \cap \mathcal{N}_{\mu}(R) \subset \mathcal{N}_{M}(Q \cap R) .
$$

\subsection{Relatively quasiconvex subgroups}

We follow the approach to relatively hyperbolic groups as developed by Hruska [7].

Definition 10 (Relative Hyperbolicity) A group $G$ is relatively hyperbolic with respect to a finite collection of subgroups $\mathbb{P}$ if $G$ acts properly discontinuously and by isometries on a proper and geodesic $\delta$-hyperbolic space $X$ with the following property: $X$ has a $G$-equivariant collection of pairwise disjoint horoballs whose union is an open set $U, G$ acts cocompactly on $X \backslash U$, and $\mathbb{P}$ is a set of representatives of the conjugacy classes of parabolic subgroups of $G$.

Throughout the rest of the paper, $G$ is a relatively hyperbolic group acting on a proper and geodesic $\delta$-hyperbolic space $X$ with a $G$-equivariant collection of horoballs satisfying all conditions of Definition 10. As before, we fix a basepoint $x_{0} \in X \backslash U$, identify each element $g$ of $G$ with $g x_{0} \in X$ and let $\operatorname{dist}\left(g_{1}, g_{2}\right)$ denote $\operatorname{dist}\left(g_{1} x_{0}, g_{2} x_{0}\right)$ for $g_{1}, g_{2} \in G$.

Lemma 11 Bowditch [4, Lemma 6.4] (Cocompact actions of parabolic subgroups on thick horospheres) Let $B$ be a horoball of $X$ with $G$-stabilizer $P$. For any $M>0, P$ acts cocompactly on $\mathcal{N}_{M}(B) \cap(X \backslash U)$.

Lemma 12 (Parabolic approximation) Let $Q$ be a subgroup of $G$ and let $\mu>0$ be a real number. There is a constant $M=M(Q, \mu)$ with the following property. If $P$ is a maximal parabolic subgroup of $G$ stabilizing a horoball $B$, and $\{1, q\} \subset Q \cap \mathcal{N}_{\mu}(B)$ then there is $p \in Q \cap P$ such that $\operatorname{dist}(p, q)<M$.

Proof By Lemma $11, \operatorname{dist}(q, P)<M_{1}$ for some constant $M_{1}=M_{1}(Q, P)$. Then Lemma 9 implies that $\operatorname{dist}(q, Q \cap P)<M_{2}$ where $M_{2}=N\left(Q, P, M_{1}\right)$. Since $B$ is a horoball at distance less than $\mu$ from 1 , there are only finitely many possibilities for $B$ and hence for the subgroup $P$. Let $M$ the maximum of all $N(Q, P, \mu)$ among the possible $P$.

Definition 13 (Relatively quasiconvex subgroup) A subgroup $Q$ of $G$ is relatively quasiconvex if there is $\mu \geq 0$ such that for any geodesic $c$ in $X$ with endpoints in $Q$, $c \cap(X \backslash U) \subset N_{\mu}(Q)$. 
The choice of horoballs turns out not to make a difference.

Proposition 14 [7] If $Q$ is relatively quasiconvex in $G$ then for any $L \geq 0$ there is $\mu \geq 0$ such that for any geodesic $c$ in $X$ with endpoints in $Q, c \cap \mathcal{N}_{L}(X \backslash U) \subset N_{\mu}(Q)$.

\section{A lemma on Gromov's inner product}

Let $Q$ and $R$ be relatively quasiconvex subgroups with compatible parabolic subgroups, and let $H$ be a finite index subgroup of $Q \cap R$.

Let $Q^{\prime}$ and $R^{\prime}$ be subgroups of $Q$ and $R$ respectively such that $Q^{\prime} \cap R^{\prime}=H$. Let $g \in Q^{\prime} R^{\prime}$ (or $g \in R^{\prime} Q^{\prime}$ ) such that $g \notin H$. Suppose $g=q r$ (or $g=r q$ ) with $q \in Q^{\prime}$, $r \in R^{\prime}$ and such that $\operatorname{dist}(1, q)+\operatorname{dist}(1, r)$ is minimal among all such products.

Lemma 15 Suppose that there exists $a \in H$ and a point $p$ at distance at most $A$ from the geodesic segment $[1, g]$ so that $\operatorname{dist}(p, q a) \leq B$. Then

$$
\operatorname{dist}(1, q)+\operatorname{dist}(1, r) \leq \operatorname{dist}(1, g)+2 A+2 B .
$$

Proof Let $p^{\prime} \in[1, g]$ be such that $\operatorname{dist}\left(p, p^{\prime}\right)<A$. Then

$$
\begin{aligned}
\operatorname{dist}(1, q a)+\operatorname{dist}\left(1, a^{-1} r\right) & \leq \operatorname{dist}\left(1, p^{\prime}\right)+\operatorname{dist}\left(p^{\prime}, q a\right)+\operatorname{dist}\left(q a, p^{\prime}\right)+\operatorname{dist}\left(p^{\prime}, g\right) \\
& \leq \operatorname{dist}(1, g)+2 A+2 B .
\end{aligned}
$$

Since $g$ can be written as $(q a)\left(a^{-1} r\right)$, the minimality assumption implies $\operatorname{dist}(1, q)+$ $\operatorname{dist}(1, r) \leq \operatorname{dist}(1, g)+2 A+2 B$.

Lemma 16 (Gromov's inner product is bounded) There is a constant $K=K(Q, R, H)$ with the following property:

$$
\operatorname{dist}(1, q)+\operatorname{dist}(1, r) \leq \operatorname{dist}(1, g)+K .
$$

Proof Constants which depend only on $Q, R, H$ and $\delta$ are denoted by $M_{i}$, the index counts positive increments of the constant during the proof. Suppose $g=q r$, the other case being symmetric. The constant $K$ of the statement corresponds to $M_{13}$.

Consider a triangle $\Delta$ with vertices $1, q, g$. Let $p \in[1, q]$ be a center of $\Delta$, ie the $\delta$-neighborhood of $p$ intersects all sides of $\Delta$.

Suppose that $p \in X \backslash U$. Then $\operatorname{dist}(p, Q)$, $\operatorname{dist}(p, q R) \leq M_{1}$ by relative quasiconvexity of $Q$ and $R$. By Lemma 9, there exists $a \in Q \cap R$ so that $\operatorname{dist}(p, q a) \leq M_{2}$. Since $H$ 


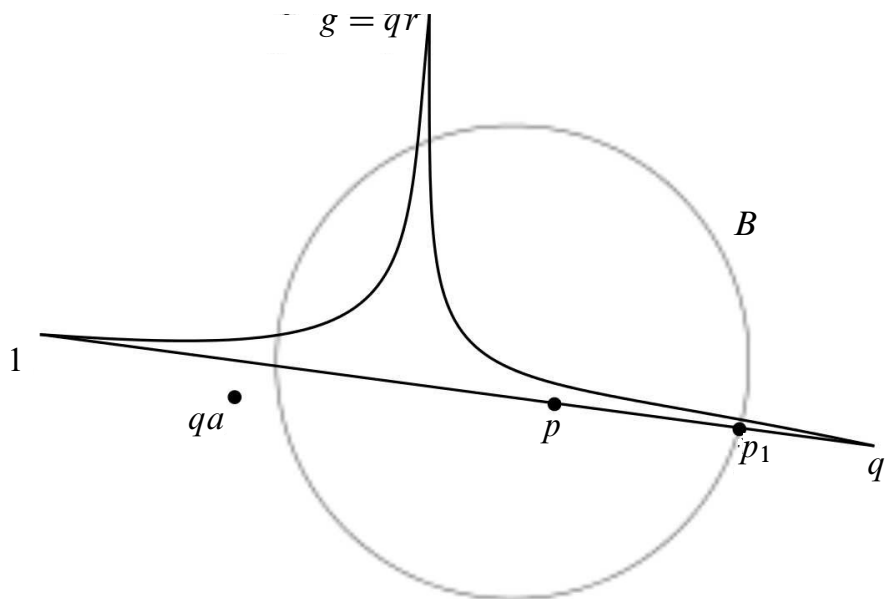

Figure 1

is a finite index subgroup of $Q \cap R$, there is $b \in H$ such that $\operatorname{dist}(p, q b) \leq M_{3}$. By Lemma $15, \operatorname{dist}(1, q)+\operatorname{dist}(1, r) \leq \operatorname{dist}(1, g)+2 M_{3}+2 \delta$.

Suppose instead that $p$ is in a horoball $B$, whose stabilizer is $P$. We can assume $\operatorname{dist}(q, B) \leq M_{8}$. Indeed, let $p_{1}$ be the entrance point of the geodesic $[q, 1]$ in $B$; then $\operatorname{dist}\left(p_{1}, Q\right)<M_{4}$ by quasiconvexity of $Q$. Notice that $\operatorname{dist}\left(p_{1},[q, g]\right)$ is at most $2 \delta$ since $p$ is a center of $\Delta$ and $p_{1} \in[q, p]$ (consider a triangle with vertices $p, q, p^{\prime}$ for $p^{\prime} \in[q, g]$ so that $\left.d\left(p, p^{\prime}\right) \leq \delta\right)$. By quasiconvexity of $R$, there is $p_{2} \in[q, g]$ such that $\operatorname{dist}\left(p_{1}, p_{2}\right), \operatorname{dist}\left(p_{2}, q R\right)<M_{5}$. Lemma 9 implies there is $a \in Q \cap R$ such that $\operatorname{dist}\left(q a, p_{1}\right), \operatorname{dist}\left(q a, p_{2}\right)<M_{6}$. Since $H$ is a finite index subgroup of $Q \cap R$, there is $b \in H$ such that $\operatorname{dist}\left(q b, p_{1}\right), \operatorname{dist}\left(q b, p_{2}\right)<M_{7}$. Since $g$ can be written as $(q b)\left(b^{-1} r\right)$, by minimality we have

$$
\begin{aligned}
\operatorname{dist}\left(1, p_{1}\right)+\operatorname{dist}\left(p_{1}, q\right)+ & \operatorname{dist}\left(q, p_{2}\right)+\operatorname{dist}\left(p_{2}, g\right) \\
& =\operatorname{dist}(1, q)+\operatorname{dist}(1, g) \\
& \leq \operatorname{dist}(1, q b)+\operatorname{dist}\left(1, b^{-1} r\right) \\
& =\operatorname{dist}\left(1, p_{1}\right)+\operatorname{dist}\left(p_{1}, q b\right)+\operatorname{dist}\left(q b, p_{2}\right)+\operatorname{dist}\left(p_{2}, g\right),
\end{aligned}
$$

and therefore

$$
\begin{aligned}
2 \operatorname{dist}(q, B) & =2 \operatorname{dist}\left(p_{1}, q\right) \\
& \leq \operatorname{dist}\left(p_{1}, q\right)+\operatorname{dist}\left(q, p_{2}\right)+\operatorname{dist}\left(p_{1}, p_{2}\right) \\
& \leq \operatorname{dist}\left(p_{1}, q b\right)+\operatorname{dist}\left(q b, p_{2}\right)+\operatorname{dist}\left(p_{1}, p_{2}\right) \\
& \leq 2 M_{8} .
\end{aligned}
$$


Since $Q$ and $R$ have compatible parabolic subgroups, assume $Q \cap q^{-1} P q \leq R \cap q^{-1} P q$, the other case being symmetric. By quasiconvexity of $Q$, there is $q_{1} \in Q$ at distance $M_{9}$ from the entrance point of $[1, q]$ in $B$. In particular, the distance from $q_{1}$ to $[1, g]$ is at most $M_{10}$. Applying the parabolic approximation lemma to $\left\{1, q^{-1} q_{1}\right\} \subset$ $Q \cap \mathcal{N}_{M_{10}}\left(q^{-1} B\right)$, there is an element $a \in Q \cap q^{-1} P q$ such that $\operatorname{dist}\left(q a, q_{1}\right) \leq M_{11}$. Since $Q \cap q^{-1} P q \leq R \cap q^{-1} P q$ it follows that $a \in Q \cap R$. Since $H$ is finite index in $Q \cap R$, by increasing the constant we can assume that $a \in H$ and $\operatorname{dist}\left(q a, q_{1}\right) \leq M_{12}$. Then Lemma 15 implies

$$
\operatorname{dist}(1, q)+\operatorname{dist}(1, r) \leq \operatorname{dist}(1, g)+M_{13} .
$$

\section{Proof of Theorem 2}

Let $Q$ and $R$ be relatively quasiconvex subgroups with compatible parabolic subgroups, and let $H$ be a finite index subgroups of $Q \cap R$.

Let $K=K(Q, R, H)$ be the constant of Lemma 16. Let $M$ be large enough so that $M>k, \lambda^{\prime} \mu^{\prime}$ where $k, \lambda^{\prime}$ and $\mu^{\prime}$ are as in Lemma 8 for $\lambda=1, \mu=K$.

Let $Q^{\prime}$ and $R^{\prime}$ be subgroups satisfying the hypothesis of the theorem, in particular $Q^{\prime} \cap R^{\prime}=H$. Consider $1 \neq g \in Q^{\prime} * Q^{\prime} \cap R^{\prime} R^{\prime}$ and suppose that $g \notin Q^{\prime} \cap R^{\prime}$. Then $g=g_{1} \ldots g_{n}$ where the $g_{i}$ 's are alternatively elements of $Q^{\prime} \backslash Q^{\prime} \cap R^{\prime}$ and $R^{\prime} \backslash Q^{\prime} \cap R^{\prime}$. Moreover, assume that this product is minimal in the sense that $\sum \operatorname{dist}\left(1, g_{i}\right)$ is minimal among all such products describing $g$.

Lemma 17 For each $i$, let $h_{i}=g_{1} \ldots g_{i}$. Then the concatenation $\alpha=\alpha_{1} \cdots \alpha_{n-1}$ of geodesics $\alpha_{i}$ from $h_{i}$ to $h_{i+1}$ is an $M$-local $(1, K)$-quasigeodesic.

Proof By the choice of $Q^{\prime}$ and $R^{\prime}$ each segment $\alpha_{i}$ has length at least $M$. Let $x \in\left[h_{i-1}, h_{i}\right]$ and $y \in\left[h_{i}, h_{i+1}\right]$. By Lemma 16 , we have

$$
\begin{aligned}
\operatorname{dist}\left(h_{i-1}, x\right)+\operatorname{dist} & (x, y)+\operatorname{dist}\left(y, h_{i+1}\right) \geq \operatorname{dist}\left(h_{i-1}, h_{i+1}\right) \\
\geq & \operatorname{dist}\left(h_{i-1}, h_{i}\right)+\operatorname{dist}\left(h_{i}, h_{i+1}\right)-K \\
= & \operatorname{dist}\left(h_{i-1}, x\right)+\operatorname{dist}\left(x, h_{i}\right)+\operatorname{dist}\left(h_{i}, y\right)+\operatorname{dist}\left(y, h_{i+1}\right)-K .
\end{aligned}
$$

Therefore $\operatorname{dist}(x, y)+K \geq \operatorname{dist}\left(x, h_{i}\right)+\operatorname{dist}\left(h_{i}, y\right)$.

Since $M>k$, Lemma 8 implies that $\alpha$ is a $\left(\lambda^{\prime}, \mu^{\prime}\right)$-quasigeodesic. Since $M>\lambda^{\prime} \mu^{\prime}$, it follows that $\alpha$ has different endpoints. Therefore we have shown that the map $Q^{\prime} * Q^{\prime} \cap R^{\prime} R^{\prime} \rightarrow G$ is injective. 
It is left to prove that if $Q^{\prime}$ and $R^{\prime}$ are relatively quasiconvex, then $\left\langle Q^{\prime}, R^{\prime}\right\rangle$ is relatively quasiconvex. Let $g \in\langle Q \cap R\rangle$ and let $\gamma$ be a geodesic from 1 to $g$. Since $H$ is quasiconvex, if $g \in H$ then $\gamma \cap(X \backslash U)$ is uniformly close to $H$ and hence to $\langle Q \cap R\rangle$. Suppose that $g \notin H$. By Lemma 7 (Morse Lemma), any $\left(\lambda^{\prime}, \mu^{\prime}\right)$-quasigeodesic is at Hausdorff distance at most $L$ from any geodesic between its endpoints. In particular, $\gamma \cap(X \backslash U) \subseteq \mathcal{N}_{L}(\alpha) \cap(X \backslash U)$ where $\alpha$ is the quasigeodesic constructed above. It is enough to show that $\alpha \cap \mathcal{N}_{L}(X \backslash U)$ is contained in $\mathcal{N}_{\mu}\left(\left\langle Q^{\prime} \cup R^{\prime}\right\rangle\right)$. Let $p \in \alpha \cap \mathcal{N}_{L}(X \backslash U)$ and let $i$ be so that $p \in\left[h_{i}, h_{i+1}\right] \cap N_{L}(X \backslash U)$. Assume $g_{i+1} \in Q^{\prime}$, the other case being symmetric. As $Q^{\prime}$ is relatively quasiconvex and in view of Proposition 14, there is a constant $\mu$ so that $p \in \mathcal{N}_{\mu}\left(h_{i} Q^{\prime}\right) \subseteq \mathcal{N}_{\mu}\left(\left\langle Q^{\prime} \cup R^{\prime}\right\rangle\right)$ (as $h_{i} \in\left\langle Q^{\prime} \cup R^{\prime}\right\rangle$ ).

\section{Separability of double cosets}

We now show Corollary 6. Suppose that all quasiconvex subgroups of $G$ are separable. Let $Q$ and $R$ be quasiconvex subgroups with compatible parabolic subgroups. Let $g \in G$ and suppose that $g \notin Q R$. We follow an argument described in Minasyan [11] and Yang [14].

Let $K=K(Q, R, Q \cap R)$ be the constant of Lemma 16. As in the proof of Theorem 2, let $M$ be large enough so that $M>k, \lambda^{\prime} \mu^{\prime}$ where $k, \lambda^{\prime}$ and $\mu^{\prime}$ are as in Lemma 8 for $\lambda=1, \mu=K$. In addition, assume that

$$
M>\lambda^{\prime} \operatorname{dist}(1, g)+\lambda^{\prime} \mu^{\prime} .
$$

Lemma 18 There are finite index subgroups $Q^{\prime}$ and $R^{\prime}$ of $Q$ and $R$ respectively such that $g \notin Q\left\langle Q^{\prime}, R^{\prime}\right\rangle R$.

Proof Since $Q \cap R$ is separable, there are finite index subgroups $Q^{\prime}$ and $R^{\prime}$ of $Q$ and $R$ respectively, such that $Q^{\prime} \cap R^{\prime}=Q \cap R$ and $\operatorname{dist}(1, f) \geq 2 M$ for any $f$ in $Q^{\prime} \backslash Q^{\prime} \cap R^{\prime}$ or $R^{\prime} \backslash Q^{\prime} \cap R^{\prime}$. By Theorem $2\left\langle Q^{\prime} \cup R^{\prime}\right\rangle$ is a quasiconvex subgroup of $G$ isomorphic to $Q^{\prime} * Q \cap R R^{\prime}$.

Suppose that $g \in Q\left\langle Q^{\prime}, R^{\prime}\right\rangle R$. Since $g \notin Q R$ it follows that $g=g_{1} \ldots g_{2 n}$ where $g_{1} \in Q, g_{2 n} \in R, g_{2 i+1} \in Q^{\prime} \backslash Q \cap R, g_{2 i} \in R^{\prime} \backslash Q \cap R$, and $n \geq 2$. Assume that this product is minimal in the sense that $\sum \operatorname{dist}\left(1, g_{i}\right)$ is minimal among all such products describing $g$.

For each $i$, let $h_{i}=g_{1} \ldots g_{i}$; let $\alpha_{i}$ be a geodesic from $h_{i}$ to $h_{i+1}$. By the choice of $Q^{\prime}$ and $R^{\prime}$ each segment $\alpha_{i}$ has length at least $2 M$ except $\alpha_{1}$ and $\alpha_{2 n-1}$. 
Notice that $g_{2} \cdots g_{2 n-1}$ represents an element of $Q^{\prime} * Q \cap R R^{\prime}$ and such product is minimal in the sense of the previous section, so that by Lemma 17 the concatenation $\alpha_{2} \cdots \alpha_{2 n-1}$ is an $M$-local $(1, K)$-quasigeodesic. Minimality of $g_{1} \ldots g_{2 n}$ and Lemma 16 imply that the concatenations $\alpha_{1} \alpha_{2}$ and $\alpha_{2 n-1} \alpha_{2 n}$ are $M$-local $(1, K)-$ quasigeodesics. Since $\alpha_{2}$ and $\alpha_{2 n-1}$ have both length at least $2 M$, it follows that the concatenation $\alpha=\alpha_{1} \cdots \alpha_{2 n}$ an $M$-local $(1, K)$-quasigeodesic.

By Lemma 8, it follows that $\alpha$ is a $\left(\lambda^{\prime}, \mu^{\prime}\right)$-quasigeodesic between 1 and $g$. It follows that $\operatorname{dist}(1, g) \geq 4 M / \lambda^{\prime}-\mu^{\prime}$; this is a contradiction with Equation (1) above.

Since $Q^{\prime}$ and $R^{\prime}$ are of finite index, there are $q_{1}, \ldots, q_{k} \in Q$ and $r_{1}, \ldots, r_{m} \in R$ such that

$$
Q\left\langle Q^{\prime}, R^{\prime}\right\rangle R=\bigcup_{q_{i}, r_{j}} q_{i}\left\langle Q^{\prime}, R^{\prime}\right\rangle r_{j}
$$

Since $\left\langle Q^{\prime}, R^{\prime}\right\rangle$ is quasiconvex, it is closed in the profinite topology. It follows that $Q\left\langle Q^{\prime}, R^{\prime}\right\rangle R$ is a finite union of closed sets. Therefore $Q\left\langle Q^{\prime}, R^{\prime}\right\rangle R$ is a closed set in the profinite topology containing $Q R$ and such that $g \notin Q\left\langle Q^{\prime}, R^{\prime}\right\rangle R$. Since $g$ was an arbitrary element of $g \in G$ not in $Q R$, it follows that $Q R$ is closed in the profinite topology of $G$.

\section{References}

[1] I Agol, D Groves, J Manning, The virtual Haken conjecture arXiv: math.GT/1204.2810

[2] I Agol, D D Long, A W Reid, The Bianchi groups are separable on geometrically finite subgroups, Ann. of Math. 153 (2001) 599-621 MR1836283

[3] M Baker, D Cooper, A combination theorem for convex hyperbolic manifolds, with applications to surfaces in 3-manifolds, J. Topol. 1 (2008) 603-642 MR2417445

[4] B H Bowditch, Relatively hyperbolic groups, Preprint, Southampton

[5] M Coornaert, T Delzant, A Papadopoulos, Géométrie et théorie des groupes, Lecture Notes in Mathematics 1441, Springer, Berlin (1990) MR1075994

[6] R Gitik, Ping-pong on negatively curved groups, J. Algebra 217 (1999) 65-72 MR1700476

[7] G C Hruska, Relative hyperbolicity and relative quasiconvexity for countable groups, Alg. Geom. Topol. 10 (2010) 1807-1856 MR2684983

[8] D D Long, A W Reid, The fundamental group of the double of the figure-eight knot exterior is GFERF, Bull. London Math. Soc. 33 (2001) 391-396 MR1832550

[9] D D Long, A W Reid, On subgroup separability in hyperbolic Coxeter groups, Geom. Dedicata 87 (2001) 245-260 MR1866851 
[10] E Martínez-Pedroza, Combination of quasiconvex subgroups of relatively hyperbolic groups, Groups Geom. Dyn. 3 (2009) 317-342 MR2486802

[11] A Minasyan, Separable subsets of GFERF negatively curved groups, J. Algebra 304 (2006) 1090-1100 MR2264291

[12] D T Wise, Subgroup separability of the figure 8 knot group, Topology 45 (2006) 421-463 MR2218750

[13] D T Wise, Research announcement: the structure of groups with a quasiconvex hierarchy, Electron. Res. Announc. Math. Sci. 16 (2009) 44-55 MR2558631

[14] W Yang, Combing fully quasiconvex subgroups and its applications arXiv: math.GT/1205. 2994

Department of Mathematics and Statistics, Memorial University

Saint John's, Newfoundland, Canada A1C 5S7

Mathematical Institute, University of Oxford

24-29 St GIles', Oxford OX1 3LB, UK

emartinezped@mun.ca, sisto@maths.ox.ac.uk

http://www.math.mun.ca/ emartinezped/,

http://people.maths.ox.ac.uk/sisto/

Received: 26 March 2012 Revised: 27 June 2012 